ISSN 1855-3966 (printed edn.), ISSN 1855-3974 (electronic edn.)

ARS MATHEMATICA CONTEMPORANEA 19 (2020) 155-171

https://doi.org/10.26493/1855-3974.2286.ece

(Also available at http://amc-journal.eu)

\title{
Oriented area as a Morse function on polygon spaces*
}

\author{
Daniil Mamaev ${ }^{\dagger}$ \\ Chebyshev Laboratory, St. Petersburg State University, \\ 14th Line V.O., 29, Saint Petersburg 199178 Russia
}

Received 20 March 2020, accepted 16 July 2020, published online 13 November 2020

\begin{abstract}
We study polygon spaces arising from planar configurations of necklaces with some of the beads fixed and some of the beads sliding freely. These spaces include configuration spaces of flexible polygons and some other natural polygon spaces. We characterise critical points of the oriented area function in geometric terms and give a formula for the Morse indices. Thus we obtain a generalisation of isoperimetric theorems for polygons in the plane.
\end{abstract}

Keywords: Flexible polygons, configuration spaces, Morse index, critical points.

Math. Subj. Class. (2020): 58K05, 52B60

\section{Preliminaries: necklaces, configuration spaces, and the oriented area function}

Suppose one has a closed string with a number of labelled beads, a necklace. Some of the beads are fixed and some can slide freely (although the beads never pass through one another). Having the necklace in hand, one can try to put it on the plane in such a way that the string is strained between every two consecutive beads. We will call this a (strained planar) configuration of the necklace. The space of all configurations (up to rotations and translations) of a given necklace, called the configuration space of the necklace, together with the oriented area function on it is the main object of the present paper.

Let us now be precise. Given a tuple $\left(n_{1}, \ldots, n_{k}\right)$ of positive integers and a tuple $\left(L_{1}, \ldots, L_{k}\right)$ of positive reals, we define a necklace $\mathbf{N}$ to be a tuple $\left(\left(n_{1}, L_{1}\right), \ldots,\left(n_{k}, L_{k}\right)\right)$ interpreted as follows:

\footnotetext{
* I am deeply indebted to Gaiane Panina for posing the problem and supervising my research. I am also thankful to Joseph Gordon and Alena Zhukova for fruitful discussions and to Nathan Blacher for his valuable comments on the linguistic quality of the paper.

${ }^{\dagger}$ The research is supported by «Native towns», a social investment program of PJSC «Gazprom Neft».

E-mail address: dan.mamaev@gmail.com (Daniil Mamaev)
} 
- the necklace has the total of $n=n(\mathbf{N})=n_{1}+\cdots+n_{k}$ beads on it;

- $k=k(\mathbf{N})$ of the beads are fixed and numbered by the index $j=1, \ldots, k$ in counterclockwise order, the index $j$ is considered to be cyclic (that is, $j=6 k+5$ is the same as $j=5)$;

- there are $\left(n_{j}-1\right)$ freely sliding beads between the $j$-th and the $(j+1)$-th fixed bead;

- the total length of the string is $L=L(\mathbf{N})=L_{1}+\cdots+L_{k}$;

- the length of the string between the $j$-th and the $(j+1)$-th fixed bead is equal to $L_{j}$.

We fix some notation concerning polygons.

- A planar $n$-gon is a collection of $n$ (labelled) points (called vertices) $\left(p_{1}, \ldots, p_{n}\right)$ in the Euclidean plane $\mathbb{R}^{2}$. Note that all kinds of degenerations, including selfintersection and collision of vertices, are allowed.

- The space of all planar n-gons Poly $_{n}$ is thus just $\left(\mathbb{R}^{2}\right)^{n}$.

- The edges of a polygon $P=\left(p_{1}, \ldots, p_{n}\right)$ are the segments $p_{i} p_{i+1}$ for $i=1, \ldots, n$, the length of the $i$-th edge is $l_{i}=l_{i}(P)=\left|p_{i} p_{i+1}\right|$. Note that the index $i=1, \ldots, n$ is cyclic (that is, $i=10 n+3$ is the same as $i=3$ ).

To avoid messy indices, we introduce some additional notation associated with a necklace $\mathbf{N}=\left(\left(n_{1}, L_{1}\right), \ldots,\left(n_{k}, L_{k}\right)\right)$ (see Figure 1 for an example). For index $j=1, \ldots, k$

- denote by $j^{*}$ the set of indices corresponding to the $j$-th piece of $\mathbf{N}$ :

$$
j^{*}=\left\{n_{1}+\cdots+n_{j-1}+1, \ldots, n_{1}+\cdots+n_{j}\right\}
$$

- define a function $\mathcal{L}_{j}:$ Poly $_{n} \rightarrow \mathbb{R}$, the total length of the edges of a polygon corresponding to the $j$-th piece of $\mathbf{N}$, that is

$$
\mathcal{L}_{j}(P)=\sum_{i \in j^{*}} l_{i}(P) .
$$

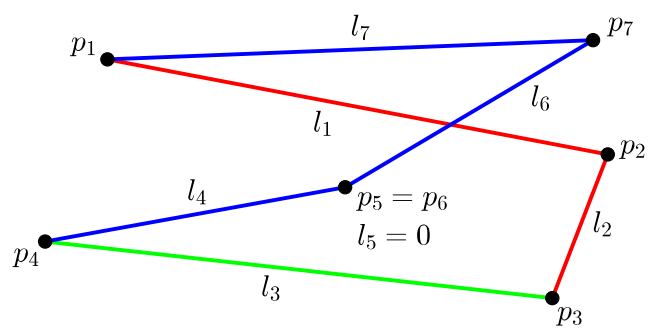

$$
\begin{aligned}
& 1^{*}=\{1,2\}, 2^{*}=\{3\}, 3^{*}=\{4,5,6,7\} \\
& s(1)=1, s(2)=3, s(3)=4 \\
& \mathcal{L}_{1}(P)=l_{1}+l_{2}=L_{1} \\
& \mathcal{L}_{2}(P)=l_{3}=L_{2} \\
& \mathcal{L}_{3}(P)=l_{4}+l_{6}+l_{7}=L_{3} .
\end{aligned}
$$

Figure 1: A configuration $P=\left(p_{1}, \ldots, p_{7}\right)$ of $\mathbf{N}=\left(\left(2, L_{1}\right),\left(1, L_{2}\right),\left(4, L_{3}\right)\right)$.

Definition 1.1. A (strained planar) configuration of a necklace $\mathbf{N}=\left(\left(n_{1}, L_{1}\right), \ldots,\left(n_{k}, L_{k}\right)\right)$ is a polygon $P \in$ Poly $_{n}$ with $\mathcal{L}_{j}(P)=L_{j}$ for all $j=1, \ldots, k$. 
All configurations of a necklace $\mathbf{N}=\left(\left(n_{1}, L_{1}\right), \ldots,\left(n_{k}, L_{k}\right)\right)$ modulo translations and rotations form a space $\mathcal{M}(\mathbf{N})=\mathcal{M}\left(\left(n_{1}, L_{1}\right), \ldots,\left(n_{k}, L_{k}\right)\right)$ called configuration space of the necklace $\mathbf{N}$. More formally,

- Consider all the strained planar configurations of $\mathbf{N}=\left(\left(n_{1}, L_{1}\right), \ldots,\left(n_{k}, L_{k}\right)\right)$ :

$$
\widetilde{\mathcal{M}}(\mathbf{N})=\left\{P \in \text { Poly }_{n}: \mathcal{L}_{j}(P)=L_{j} \text { for } j=1, \ldots, k\right\} .
$$

- The group Iso $+\left(\mathbb{R}^{2}\right)$ of orientation-preserving isometries of the Euclidean plane $\mathbb{R}^{2}$ acts diagonally on the space of all planar $n$-gons Poly $_{n}=\left(\mathbb{R}^{2}\right)^{n}$.

- The configuration space of the necklace $\mathbf{N}$ is the space of orbits:

$$
\mathcal{M}(\mathbf{N})=\widetilde{\mathcal{M}}(\mathbf{N}) / \mathrm{IsO}_{+}\left(\mathbb{R}^{2}\right)
$$

Definition 1.2. The oriented area $\mathcal{A}$ of an $n$-gon $P=\left(\left(x_{1}, y_{1}\right), \ldots,\left(x_{n}, y_{n}\right)\right) \in\left(\mathbb{R}^{2}\right)^{n}$ is defined to be

$$
\mathcal{A}(P)=\frac{1}{2}\left|\begin{array}{ll}
x_{1} & x_{2} \\
y_{1} & y_{2}
\end{array}\right|+\frac{1}{2}\left|\begin{array}{ll}
x_{2} & x_{3} \\
y_{2} & y_{3}
\end{array}\right|+\cdots+\frac{1}{2}\left|\begin{array}{ll}
x_{n} & x_{1} \\
y_{n} & y_{1}
\end{array}\right| .
$$

The oriented area is preserved by the action of $\mathrm{IsO}_{+}\left(\mathbb{R}^{2}\right)$ and thus gives rise to a welldefined continuous function on $\mathcal{M}(\mathbf{N})$ for all the necklaces $\mathbf{N}$. We will denote these functions by the same letter $\mathcal{A}$. The study of critical points (i.e. the solutions of $d \mathcal{A}(P)=0$ ) of $\mathcal{A}: \mathcal{M}(\mathbf{N}) \rightarrow \mathbb{R}$ is the subject of the present paper.

The paper is organised as follows. In Section 2 we review previously studied extreme cases: polygonal linkages (the 'all beads are fixed' case) and polygons with fixed perimeter (the 'one bead is fixed' case, which is clearly the same as 'none of the beads are fixed' case). In Section 3 we discuss the regularity properties of configuration spaces of necklaces. In the subsequent sections we study the non-singular part of the configuration space. In Section 4 we give a geometric description of critical points of the oriented area in the general case (Theorem 4.1) and deduce a formula for their Morse indices (Theorem 4.2). In Section 5 the auxiliary Lemmata 4.3 and 4.4 concerning orthogonality of certain spaces with respect to the Hessian form of the oriented area function are proven. In Section 6 we discuss the 'two consecutive beads are fixed' case and give a proof of Lemma 4.5.

\section{An overview of existing results}

\subsection{Configuration spaces of polygonal linkages}

In the notation of the present paper these are the spaces $\mathcal{M}\left(\left(1, l_{1}\right), \ldots,\left(1, l_{n}\right)\right)$, i.e. the spaces $\mathcal{M}(\mathbf{N})$ for necklaces $\mathbf{N}$ with all beads being fixed. These spaces are studied in many aspects (see e.g. [1] or [2] for a thorough survey). On the side of studying the oriented area on these spaces, the first general fact about its critical points was noticed by Thomas Banchoff (unpublished), reproved by Khimshiashvili and Panina [5] (their technique required some non-degeneracy assumptions) and then proved again by Leger [8] in full generality.

Theorem 2.1 (Critical configurations in the 'all beads are fixed' case; Bunchoff, Khimshiashvili, Leger, and Panina). Let $\mathbf{N}$ be a necklace with all the beads fixed. Then a polygon $P \in \mathcal{M}_{\text {sm }}(\mathbf{N})$ is a critical point of $\mathcal{A}$ if and only if it is cyclic (i.e. inscribed in a circle). 
After describing critical points, the following natural question arises: are these critical points Morse (i.e. whether $\operatorname{Hess}_{P} \mathcal{A}$, the Hessian of $\mathcal{A}$ at $P$, is a non-degenerate bilinear form on $T_{P} \mathcal{M}(\mathbf{N})$ ) and if they are, what is the Morse index (the maximal dimension of a subspace on which $\operatorname{Hess}_{P} \mathcal{A}$ is negative definite). The state-of-the-art answer to this question requires some more notation (see Figure 2 for an example).

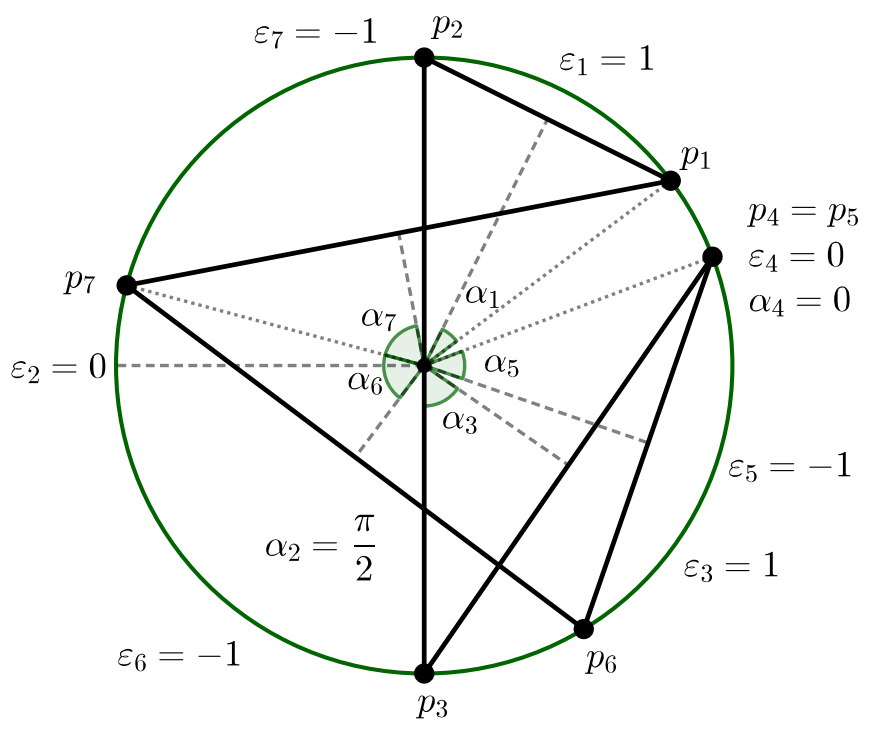

Figure 2: Notation for a cyclic polygon.

Definition 2.2. Let $P$ be a cyclic $n$-gon, $o$ be the circumcentre of $P$, and $i \in\{1, \ldots, n\}$.

- The central half-angle of the $i$-th edge of $P$ is

$$
\alpha_{i}(P)=\frac{\left|\angle p_{i} o p_{i+1}\right|}{2} \in[0, \pi / 2] .
$$

- The orientation of the $i$-th edge of $P$ is

$$
\varepsilon_{i}(P)=\left\{\begin{aligned}
1, & \text { if } \angle p_{i} o p_{i+1} \in(0, \pi) \\
0, & \text { if } \angle p_{i} o p_{i+1} \in\{0, \pi\} \\
-1, & \text { if } \angle p_{i} o p_{i+1} \in(-\pi, 0)
\end{aligned}\right.
$$

We will denote by $\mathcal{C}_{n}$ the configuration space of cyclic $n$-gons with at least three vertices. More precisely,

$$
\mathcal{C}_{n}=\left\{P \in\left(\mathbb{R}^{2}\right)^{n}: \begin{array}{l}
P \text { is a cyclic polygon; } \\
\text { AffineHull }(P)=\mathbb{R}^{2}
\end{array}\right\} / \operatorname{Iso}_{+}\left(\mathbb{R}^{2}\right) .
$$

For $P \in \mathcal{C}_{n}$ denote by $\Omega_{P}$ its circumscribed circle, by $o_{P}$ its circumcentre, and by $R_{P}$ the radius of $\Omega_{P}$. 
Definition 2.3. Let $P$ be a cyclic polygon with at least three distinct vertices. It is called admissible if no edge of $P$ passes through its circumcentre $o$. In this case its winding number $w_{P}=w(P, o)$ with respect to $o$ is well-defined.

Theorem 2.4 (Morse indices in the 'all beads are fixed' case; Gordon, Khimshiashvili, Panina, Teplitskaya, and Zhukova). Let $\mathbf{N}=\left(\left(1, l_{1}\right), \ldots,\left(1, l_{n}\right)\right)$ be a necklace without freely moving beads, and let $P \in \mathcal{M}_{s m}(\mathbf{N})$ be an admissible cyclic polygon. Then $P$ is a Morse point of $\mathcal{A}$ if and only if $\sum_{i=1}^{n} \varepsilon_{i} \tan \alpha_{i} \neq 0$ and in this case its Morse index is

$$
\mu_{P}(\mathcal{A})=\#\left\{i \in\{1, \ldots, n\}: \varepsilon_{i}>0\right\}-1-2 w_{P}- \begin{cases}0, & \text { if } \sum_{i=1}^{n} \varepsilon_{i} \tan \alpha_{i}>0 \\ 1, & \text { otherwise }\end{cases}
$$

The formula more or less explicitly appeared in [6, 9], and [11], but in this form, with the precise condition of being Morse, the theorem was proved only in [3]. The following definition was first given in [3].

Definition 2.5. An admissible cyclic polygon $P$ is called bifurcating if $\sum_{i=1}^{n} \varepsilon_{i} \tan \alpha_{i}=0$.

\subsection{Configuration space of $\boldsymbol{n}$-gons with fixed perimeter}

This is the space $\mathcal{M}((n, L))=\mathcal{M}(n, L)$ (for different $L$ these spaces are isomorphic, so usually $L$ is set to 1$)$. It is no secret since antiquity, that, with perimeter fixed, convex regular polygons maximise the area. All the critical points of the oriented area together with their indices were determined only in a recent paper [7] by Khimshiashvili, Panina and Siersma.

Definition 2.6. A regular star is a cyclic polygon $P$ such that all its edges are equal and have the same orientation (see (2.1)). A complete fold is a regular star $P$ with $p_{i}=p_{i+2}$ for all $i=1, \ldots, n$. It exists for even $n$ only.

Theorem 2.7 (Critical configurations and Morse indices in the 'one bead is fixed' case; Khimshiashvili, Panina, and Siersma).

(1) $\mathcal{M}(n, L)$ is homeomorphic to $\mathbb{C P}^{n-2}$.

(2) A polygon $P \in \mathcal{M}_{s m}(n, L)$ is a critical point of $\mathcal{A}$ if and only if it is a regular star.

(3) The stars with maximal winding numbers are Morse critical points of $\mathcal{A}$.

(4) Under assumption that all regular stars are Morse critical points, the Morse indices are:

$$
\mu_{P}^{n}(\mathcal{A})= \begin{cases}2 w_{P}-2, & \text { if } w_{P}<0 \\ 2 n-2 w_{P}-2, & \text { if } w_{P}>0 \\ n-2, & \text { if } P \text { is a complete fold }\end{cases}
$$

Remark 2.8. The super-index in $\mu_{P}(\mathcal{A})$ allows one to identify the domain of $\mathcal{A}$. For example, $\mu_{P}^{n}(\mathcal{A})$ is the Morse index of $\mathcal{A}: \mathcal{M}\left(n, \mathcal{L}_{1}(P)\right) \rightarrow \mathbb{R}$ at point $P$ (as in (4) of Theorem 2.7), and $\mu_{P}^{1, \ldots, 1}(\mathcal{A})$ is the Morse index of $\mathcal{A}: \mathcal{M}\left(\left(1, \mathcal{L}_{1}(P)\right), \ldots,\left(1, \mathcal{L}_{n}(P)\right)\right) \rightarrow \mathbb{R}$ at point $P$ (as in Theorem 2.4). This notation will be of much use in the proof of Theorem 4.2. 
Let us also mention an auxiliary statement proven in [7].

Lemma 2.9 (Khimshiashvili, Panina, and Siersma). Let $P$ be a regular star which is not a complete fold with $w_{P}>0$. Then $P$ is a non-degenerate local maximum on $\mathcal{C}_{n}$.

In fact, this lemma together with Theorem 2.4 and Lemma 4.3 allows one to omit the assumption in (4) of Theorem 2.7. All the critical points of the oriented area on $\mathcal{C}_{n}$ were described in a recent preprint [10] by Siersma.

\section{Singular locus of the configuration space}

Definition 3.1. Let $P$ be a configuration of a necklace $\mathbf{N}=\left(\left(n_{1}, L_{1}\right), \ldots,\left(n_{k}, L_{k}\right)\right)$. It is called non-singular if $\mathcal{L}=\left(\mathcal{L}_{1}, \ldots, \mathcal{L}_{k}\right)$ is a smooth submersion at $P$ (i.e. $\mathcal{L}$ is differentiable at $P$ and its differential $D_{P} \mathcal{L}: T_{P}$ Poly $_{n} \rightarrow T_{P} \mathbb{R}^{k}$ is a surjective linear map), otherwise it is called singular.

First we give a geometric characterisation of singular configurations. Consider a polygon $P=\left(p_{1}, \ldots, p_{n}\right)$, with $p_{i}=\left(x_{i}, y_{i}\right) \in \mathbb{R}^{2}$ and $l_{i}=\left|p_{i+1}-p_{i}\right| \neq 0$ for all $i=1, \ldots, n$. Define $\beta_{i}$ to be the oriented angle between vectors $(1,0)$ and $\left(p_{i+1}-p_{i}\right)$. Denote by $s(j)=n_{1}+\cdots+n_{j-1}+1$ the index of the $j$-th fixed bead. Then every $\mathcal{L}_{j}$ is differentiable at $P$ and the derivative of $\mathcal{L}_{j}$ with respect to $x_{i}$ and $y_{i}$ is as follows:

$$
\begin{aligned}
& \frac{\partial \mathcal{L}_{j}}{\partial x_{i}}(P)= \begin{cases}-\cos \beta_{i}, & \text { if } i=s(j) \\
\cos \beta_{i-1}-\cos \beta_{i}, & \text { if } i \in j^{*} \backslash\{s(j)\} \\
\cos \beta_{i-1}, & \text { if } i=s(j+1) \\
0, & \text { otherwise }\end{cases} \\
& \frac{\partial \mathcal{L}_{j}}{\partial y_{i}}(P)= \begin{cases}-\sin \beta_{i}, & \text { if } i=s(j) \\
\sin \beta_{i-1}-\sin \beta_{i}, & \text { if } i \in j^{*} \backslash\{(j)\} \\
\sin \beta_{i-1}, & \text { if } i=s(j+1) \\
0, & \text { otherwise }\end{cases}
\end{aligned}
$$

Definition 3.2. Let $\mathbf{N}=\left(\left(n_{1}, L_{1}\right), \ldots,\left(n_{k}, L_{k}\right)\right)$ be a necklace. An index $i \in\{1, \ldots, n(\mathbf{N})\}$ is called boundary if it is equal to $s(j)$ for some $j \in\{1, \ldots, k\}$, otherwise it is called inner.

In Figure 1 the indices 1,3,4 are boundary and the indices 2, 5, 6, 7 are inner.

Lemma 3.3. A configuration $P \in$ Poly $_{n}$ of the necklace $\mathbf{N}=\left(\left(n_{1}, L_{1}\right), \ldots,\left(n_{k}, L_{k}\right)\right)$ is singular if and only if one of the following holds:

(1) $l_{i}=0$ for some $i \in\{1, \ldots, n(\mathbf{N})\}$;

(2) $P$ fits in a straight line in such a way that $\beta_{i}=\beta_{i-1}$ for all inner indices $i$.

Proof. The first condition is equivalent to $\mathcal{L}$ being differentiable at $P$. Therefore, what is left to prove, is that for $P \in \widetilde{\mathcal{M}}(\mathbf{N})$ with no vanishing edges, the second condition holds if and only if the gradients $\operatorname{grad}_{P} \mathcal{L}_{1}, \ldots, \operatorname{grad}_{P} \mathcal{L}_{k}$ are linearly dependent.

Suppose that $\lambda_{1} \operatorname{grad}_{P} \mathcal{L}_{1}+\cdots+\lambda_{k} \operatorname{grad}_{P} \mathcal{L}_{k}=0$ is a non-trivial vanishing linear combination. If $\lambda_{j} \neq 0$, then, using formulae (3.1) and (3.2) for boundary index $s(j)$, 
we get $-\lambda_{j} \cos \beta_{s(j)}+\lambda_{j-1} \cos \beta_{s(j)-1}=0$ and $-\lambda_{j} \sin \beta_{s(j)}+\lambda_{j-1} \sin \beta_{s(j)-1}=0$. It means that points $\lambda_{j}\left(\cos \beta_{s(j)}, \sin \beta_{s(j)}\right) \neq(0,0)$ and $\lambda_{j-1}\left(\cos \beta_{s(j)-1}, \sin \beta_{s(j)-1}\right)$ coincide, which implies that $2\left(\beta_{s(j)}-\beta_{s(j)-1}\right)=0$ and $\lambda_{j-1}=\cos \left(\beta_{s(j)}-\beta_{s(j)-1}\right) \lambda_{j} \neq$ 0 . It follows then that $\lambda_{j} \neq 0$ for all $j=1, \ldots, k$, consequently, (we now use (3.1) and (3.2) for inner indices) $\beta_{i}=\beta_{i-1}$ for all inner indices $i$, meaning that $P$ is composed of straight segments of lengths $L_{1}, \ldots, L_{k}$. Taking into account previously deduced formula $2\left(\beta_{i}-\beta_{i-1}\right)=0$ for boundary $i$, we conclude that $P$ does satisfy condition (2). Reversing the above argument, we get the reverse implication.

Definition 3.4. A singular configuration $P$ of a necklace $\mathbf{N}$ is called strongly singular, if it satisfies (2) in Lemma 3.3. Otherwise it is called weakly singular.

Remark 3.5. Let $\mathbf{N}=\left(\left(n_{1}, L_{1}\right), \ldots,\left(n_{k}, L_{k}\right)\right)$ be a necklace. Then

- weakly singular configurations of $\mathbf{N}$ are, in a sense, inessential (for instance, $\mathcal{M}(\mathbf{N}$ ) is a topological manifold around them);

- if $\left(L_{1}, \ldots, L_{n}\right)$ is such that $\pm L_{1} \pm \cdots \pm L_{n} \neq 0$ for any choice of \pm (such tuples are called generic in [2]), then there are no strongly singular configurations of $\mathbf{N}$.

Together, these two facts allow to deduce some information about topology of $\mathcal{M}(\mathbf{N})$ for generic $\mathbf{N}$ from Theorems 4.1 and 4.2, but this is not the subject of the present paper.

Now let $\widetilde{\mathcal{M}}_{s m}(\mathbf{N})$ be the set of non-singular configurations of necklace $\mathbf{N}$ and $\mathcal{M}_{\text {sm }}(\mathbf{N})$ be the non-singular part of $\mathcal{M}(\mathbf{N})$ :

$$
\mathcal{M}_{s m}(\mathbf{N})=\frac{\widetilde{\mathcal{M}}_{s m}(\mathbf{N})}{\mathrm{Iso}_{+}\left(\mathbb{R}^{2}\right)}=\left\{P \in \text { Poly }_{n}: \begin{array}{l}
P \text { is a non-singular } \\
\text { configuration of } \mathbf{N}
\end{array}\right\} / \operatorname{Iso}_{+}\left(\mathbb{R}^{2}\right)
$$

If these spaces are non-empty, they are smooth manifolds. This statement generalises previous results on smoothness of configuration spaces of polygonal linkages by KapovichMillson [4] and Farber [2].

Definition 3.6. A necklace $\mathbf{N}=\left(\left(n_{1}, L_{1}\right), \ldots,\left(n_{k}, L_{k}\right)\right)$ is called realisable if for all $j=1, \ldots, k$, such that $n_{j}=1$, the inequality $2 L_{j}<L_{1}+\cdots+L_{k}$ holds.

Proposition 3.7. Let $\mathbf{N}$ be a realisable necklace. Then

(1) $\widetilde{\mathcal{M}}_{\text {sm }}(\mathbf{N})$ is a smooth $(2 n-k)$-dimensional submanifold of Poly ${ }_{n}=\mathbb{R}^{2 n}$;

(2) $\mathcal{M}_{s m}(\mathbf{N})$ is a topological manifold of dimension $2 n-k-3$ with a unique smooth structure making the quotient map $\widetilde{\mathcal{M}}_{s m}(\mathbf{N}) \rightarrow \mathcal{M}_{\text {sm }}(\mathbf{N})$ a smooth submersion;

(3) the oriented area function $\mathcal{A}$ is a smooth function on $\mathcal{M}_{s m}(\mathbf{N})$.

Proof. It follows from Lemma 3.3, that the inequalities $2 L_{j}<L_{1}+\cdots+L_{k}$ are necessary and sufficient for $\widetilde{\mathcal{M}}_{s m}(\mathbf{N})$ to be non-empty.

The first claim is clear since $\widetilde{\mathcal{M}}_{s m}(\mathbf{N})$ is locally a level of a smooth submersion $\mathcal{L}=\left(\mathcal{L}_{1}, \ldots, \mathcal{L}_{k}\right):\left(\mathbb{R}^{2}\right)^{n} \rightarrow \mathbb{R}^{k}$.

To establish the second claim, we first note that $\mathcal{M}_{s m}(\mathbf{N})$ is an orbit space of the action of 3-dimensional Lie group Iso $+\left(\mathbb{R}^{2}\right)$ on the smooth manifold $\widetilde{\mathcal{M}}_{s m}(\mathbf{N})$. Thus, it suffices to observe that the action is free and proper, which is indeed the case.

The third claim is obvious since the smooth structure on $\mathcal{M}_{s m}(\mathbf{N})$ is induced from Poly $_{n}$, and the oriented area $\mathcal{A}$ is a smooth function (cf. (1.2)) on Poly ${ }_{n}$ preserved by the action of $\mathrm{IsO}_{+}\left(\mathbb{R}^{2}\right)$. 


\section{Main results: critical configurations and their Morse indices in the general case}

The following theorem describes critical points of the oriented area on configuration spaces of necklaces. It generalises Theorem 2.1 and (2) in Theorem 2.7.

Theorem 4.1 (Critical configurations in the general case). A polygon $P \in \mathcal{M}_{s m}\left(\left(n_{1}, L_{1}\right), \ldots,\left(n_{k}, L_{k}\right)\right)$ is a critical point of $\mathcal{A}$ if and only if all of the following conditions hold:

(1) $P$ is cyclic;

(2) $l_{i}(P)=L_{j} / n_{j}$ for all $i \in j^{*}$;

(3) $\varepsilon_{i_{1}}(P)=\varepsilon_{i_{2}}(P)$ for all $i_{1}, i_{2} \in j^{*}$,

where $j^{*}$ is the set of indices corresponding to the $j$-th piece of a necklace (see (1.1)) and $\varepsilon_{i}(P)$ is the orientation of the $i$-th edge of a cyclic polygon $P$ (see (2.1)).

The proof essentially is a reformulation of geometric arguments into the language of Lagrange multipliers, so we first write partial derivatives of $\mathcal{A}$ with respect to $x_{i}$ and $y_{i}$ :

$$
\begin{aligned}
2 \cdot \frac{\partial \mathcal{A}}{\partial x_{i}}(P) & =l_{i-1} \sin \beta_{i-1}+l_{i} \sin \beta_{i} \\
2 \cdot \frac{\partial \mathcal{A}}{\partial y_{i}}(P) & =-l_{i-1} \cos \beta_{i-1}-l_{i} \cos \beta_{i} .
\end{aligned}
$$

We follow the convention $0 \cdot$ undefined $=0$ hence both sides are defined for all $P \in$ Poly $_{n}$.

Proof of Theorem 4.1. Let $P$ be a non-singular configuration of a necklace $\mathbf{N}=$ $\left(\left(n_{1}, L_{1}\right), \ldots,\left(n_{k}, L_{k}\right)\right)$. Then $P$ is a critical point of $\mathcal{A}$ if and only if there exist $\lambda_{1}, \ldots$, $\lambda_{k} \in \mathbb{R}$, such that $2 \cdot \operatorname{grad}_{P} \mathcal{A}=\lambda_{1} \operatorname{grad}_{P} \mathcal{L}_{1}+\cdots+\lambda_{k} \operatorname{grad}_{P} \mathcal{L}_{k}$.

Assume that $2 \cdot \operatorname{grad}_{P} \mathcal{A}=\lambda_{1} \operatorname{grad}_{P} \mathcal{L}_{1}+\cdots+\lambda_{k} \operatorname{grad}_{P} \mathcal{L}_{k}$. Applying formulae (3.1), (3.2), (4.1), (4.2) to an inner index $i \in j^{*}$, one deduces

$$
\begin{aligned}
l_{i-1} \sin \beta_{i-1}+l_{i} \sin \beta_{i} & =\lambda_{j}\left(\cos \beta_{i-1}-\cos \beta_{i}\right) ; \\
-l_{i-1} \cos \beta_{i-1}-l_{i} \cos \beta_{i} & =\lambda_{j}\left(\sin \beta_{i-1}-\sin \beta_{i}\right) .
\end{aligned}
$$

If $\beta_{i}=\beta_{i-1}$, then $l_{i}=l_{i-1}=0$, but $P$ is non-singular, so it cannot be the case by Lemma 3.3. The only other possibility for these equations to hold is $l_{i-1}=l_{i}$ and $\lambda_{j}=l_{i} \cot \left(\frac{\beta_{i}-\beta_{i-1}}{2}\right)$. Since we have such equations for all inner indices corresponding to $j$, we get $l_{i_{1}}=l_{i_{2}}$ for all $i_{1}, i_{2} \in j^{*}$, which implies condition (2) of the theorem. Moreover, for all $i \in j^{*} \backslash s(j)$ we get $\cot \left(\frac{\beta_{i}-\beta_{i-1}}{2}\right)=\frac{n_{j} \lambda_{j}}{L_{j}}$, therefore $\beta_{i}-\beta_{i-1}$ is the same for all $i \in j^{*} \backslash s(j)$, which implies that there is a circle $\Omega_{j}$ with centre $o_{j}$ such that conditions (2) and (3) of the theorem hold. It now remains to prove that $P$ is cyclic, i.e. $o_{j}$ is the same for all $j=1, \ldots, k$. If $P$ is a smooth point of $\mathcal{M}_{s m}\left(\left(1, l_{1}\right), \ldots,\left(1, l_{n}\right)\right) \subset \mathcal{M}_{s m}(\mathbf{N})$, in other words, if $P$ does not fit in a straight line, then we are done by Theorem 2.1. Suppose that $P$ fits in a straight line. Pick a boundary vertex $i=s(j+1)$, denote $l^{j}=L_{j} / n_{j}$, and apply formulae (3.1), (3.2), (4.1), (4.2) to $i$ :

$$
\begin{aligned}
l^{j} \sin \beta_{i-1}+l^{j+1} \sin \beta_{i} & =\lambda_{j} \cos \beta_{i-1}-\lambda_{j+1} \cos \beta_{i} ; \\
-l^{j} \cos \beta_{i-1}-l^{j+1} \cos \beta_{i} & =\lambda_{j} \sin \beta_{i-1}-\lambda_{j+1} \sin \beta_{i} .
\end{aligned}
$$


Since $P$ fits in a straight line, $2\left(\beta_{i}-\beta_{i-1}\right)=0$. If $\beta_{i-1}=\beta_{i}=\beta$, then the points $\left(l^{j}+l^{j+1}\right)(\cos \beta, \sin \beta)$ and $\left(\lambda_{j}-\lambda_{j+1}\right)(\cos (\beta+\pi / 2), \sin (\beta+\pi / 2))$ coincide which cannot be the case since $l^{j}, l^{j+1}>0$. If $\beta_{i-1}=\beta_{i}+\pi=\beta+\pi$, then the points $\left(l^{j}-l^{j+1}\right)(\cos \beta, \sin \beta)$ and $\left(\lambda_{j}+\lambda_{j+1}\right)(\cos (\beta+\pi / 2), \sin (\beta+\pi / 2))$ coincide, which implies that $l^{j}=l^{j+1}$. Since this is the case for all $j, P$ is a complete fold and thus indeed is cyclic.

Now assume that a non-singular configuration $P$ of necklace $\mathbf{N}$ satisfies conditions (1)-(3). Let $\Omega$ be its circumscribed circle with centre $o$. Denote by $\gamma_{j}$ the oriented angle $\angle p_{s(j) o p_{s(j)+1}}$ and set $\lambda_{j}=l_{i} \cot \left(\gamma_{j} / 2\right)$ for some index $i$ corresponding to $j$. Since $\gamma_{j}=\beta_{i}-\beta_{i-1}$ for inner indices $i$, equality $2 \cdot \operatorname{grad}_{P} \mathcal{A}=\lambda_{1} \operatorname{grad}_{P} \mathcal{L}_{1}+\cdots+\lambda_{k} \operatorname{grad}_{P} \mathcal{L}_{k}$ holds in all inner indices. For a boundary index $i=s(j+1)$ we can (performing rotation around $o$ ) assume that $\beta_{i-1}=0$, and what we need to check then is the following two equalities:

$$
\begin{aligned}
l^{j+1} \sin \beta_{i} & =l^{j} \cot \left(\gamma_{i-1} / 2\right)-l^{j+1} \cot \left(\gamma_{i} / 2\right) \cos \beta_{i} ; \\
-l^{j}-l^{j+1} \cos \beta_{i} & =-l^{j+1} \cot \left(\gamma_{i} / 2\right) \sin \beta_{i},
\end{aligned}
$$

Putting the origin at $o$, we note that

$$
\begin{aligned}
p_{i+1}-p_{i} & =l^{j+1} \cdot\left(\cos \beta_{i}, \sin \beta_{i}\right), \\
p_{i+1}+p_{i} & =l^{j+1} \cot \frac{\gamma_{i}}{2} \cdot\left(-\sin \beta_{i}, \cos \beta_{i}\right), \\
p_{i} & =\left(\frac{l_{j}}{2},-\frac{l_{j}}{2} \cot \frac{\gamma_{i-1}}{2}\right),
\end{aligned}
$$

and thus the desired equalities are just the coordinate manifestations of the obvious identity

$$
\frac{p_{i+1}-p_{i}}{2}-\frac{p_{i+1}+p_{i}}{2}+p_{i}=(0,0)
$$

The following theorem provides a criterion for an admissible cyclic polygon to be a Morse point of the oriented area and gives a formula for its Morse index. It generalises Theorem 2.4 and allows one to omit the assumption in (4) of Theorem 2.7.

Theorem 4.2 (Morse indices in the general case). Let $\mathbf{N}=\left(\left(n_{1}, L_{1}\right), \ldots,\left(n_{k}, L_{k}\right)\right)$ be a realisable necklace (see Definition 3.6), and $P \in \mathcal{M}_{\text {sm }}(\mathbf{N})$ be an admissible (see Definition 2.3) critical point of the oriented area $\mathcal{A}$. Then $P$ is a Morse point of $\mathcal{A}$ if and only if it is not a bifurcating polygon (see Definition 2.5). In this case its Morse index can be computed as follows:

$$
\mu_{P}(\mathcal{A})=\frac{1}{2} \sum_{j=1}^{k}\left(2 n_{j}-1\right) \cdot\left(E_{j}+1\right)-1-2 w_{P}- \begin{cases}0, & \text { if } \sum_{j=1}^{k} n_{j} E_{j} \tan A_{j}>0 \\ 1, & \text { otherwise }\end{cases}
$$

where $E_{j}=\varepsilon_{i}$ and $A_{j}=\alpha_{i}$ for some $i \in j^{*}$ (due to Theorem 4.1 this does not depend on the choice of $i$ ).

Proof. Let $P$ be as in the theorem. First, let us split the tangent space of $\mathcal{M}_{s m}(\mathbf{N})$ at the critical point $P$ into subspaces that are orthogonal with respect to the Hessian form $\operatorname{Hess}_{P} \mathcal{A}$. For this, given a polygon $P$, we introduce the following submanifolds in $\mathcal{M}_{s m}(\mathbf{N})$ : 
(1) $\mathcal{E}^{P}=\mathcal{M}_{s m}\left(\left(1, l_{1}\right), \ldots,\left(n, l_{n}\right)\right) \subset \mathcal{M}_{s m}(\mathbf{N})$ is the space of all polygons having the same edge length as $P$;

(2) $\mathcal{C}^{P}=\mathcal{M}_{s m}(\mathbf{N}) \cap \mathcal{C}$ is the subspace of cyclic polygons;

(3) $\mathcal{C}_{j}^{P}=\left\{Q \in \mathcal{M}_{s m}(\mathbf{N}): \begin{array}{c}\left(q_{s(j)}, \ldots, q_{s(j+1)}\right) \text { is cyclic } \\ q_{i}=p_{i} \text { for } i \notin j^{*} \backslash\{s(j)\}\end{array}\right\}$ for $j=1, \ldots, k$.

We will deduce the theorem from Lemmata 4.3, 4.4, and 4.5 (see Sections 5 and 6 for their proofs).

Lemma 4.3. Let $P$ be as in Theorem 4.2. Then

(1) $\mathcal{E}^{P} \subset \mathcal{M}_{s m}(\mathbf{N})$ is a smooth $(n-3)$-dimensional submanifold in a neighbourhood of $P$;

(2) $\mathcal{C}^{P} \subset \mathcal{M}_{s m}(\mathbf{N})$ is a smooth $(n-k)$-dimensional submanifold in a neighbourhood of $P$;

(3) $\mathcal{E}^{P}$ and $\mathcal{C}^{P}$ intersect transversally at $P$, i.e. $T_{P} \mathcal{M}_{s m}(\mathbf{N})=T_{P} \mathcal{E}^{P} \oplus T_{P} \mathcal{C}^{P}$;

(4) $T_{P} \mathcal{E}^{P}$ and $T_{P} \mathcal{C}^{P}$ are orthogonal with respect to the bilinear form $\operatorname{Hess}_{P} \mathcal{A}$.

One can note that none of the $\mathcal{C}_{j}^{P}$ are contained in $\mathcal{C}^{P}$. Nonetheless, from the following lemma one sees that in the first approximation they very much are.

Lemma 4.4. Let $P$ be as in Theorem 4.2. Then

(1) $\mathcal{C}_{j}^{P} \subset \mathcal{M}_{s m}(\mathbf{N})$ is a smooth $\left(n_{j}-1\right)$-dimensional submanifold in a neighbourhood of $P$;

$$
T_{P} \mathcal{C}^{P}=\bigoplus_{j=1}^{k} T_{P} \mathcal{C}_{j}^{P}
$$

(3) $T_{P} \mathcal{C}_{j}^{P}$ are pairwise orthogonal with respect to the bilinear form $\operatorname{Hess}_{P} \mathcal{A}$.

It remains to compute the Morse index of $P$ with respect to $\mathcal{A}$ on each $\mathcal{C}_{j}^{P}$.

Lemma 4.5. Suppose that $P \in \mathcal{C}_{n+1}$ is such that $l_{1}=\cdots=l_{n}=L / n$ and $\varepsilon_{i}=1$ $\left(\varepsilon_{i}=-1\right)$ for $i=1, \ldots, n$. Then $P$ is a non-degenerate local maximum (minimum) of the oriented area on $\mathcal{M}_{s m}\left((n, L),\left(1, l_{n}\right)\right) \cap \mathcal{C}_{n+1}$.

Now we are ready to prove the theorem. From Lemmata 4.3 and 4.4, $P$ is a Morse point of $\mathcal{A}$ on $\mathcal{M}_{s m}$ if and only if it is a Morse point of $\mathcal{A}$ on $\mathcal{E}^{P}$ and all of $\mathcal{C}_{j}^{P}$. Since $P$ is always a Morse point on each $\mathcal{C}_{j}^{P}$ (because by Lemma 4.5 it is a non-degenerate local extremum), it is a Morse point of $\mathcal{A}$ on $\mathcal{M}_{s m}$ if and only if it is a Morse point of $\mathcal{A}$ on $\mathcal{E}^{P}$, which is equivalent to $P$ not being bifurcating by Theorem 2.4.

Moreover, Lemmata 4.3 and 4.4 imply that if $P$ is a Morse point of $\mathcal{A}$ on $\mathcal{M}_{\text {sm }}$, then its Morse index is

$$
\mu_{P}^{n_{1}, \ldots, n_{k}}(\mathcal{A})=\mu_{P}^{\mathcal{E}^{P}}(\mathcal{A})+\mu_{P}^{\mathcal{C}^{P}}(\mathcal{A})=\mu_{P}^{1, \ldots, 1}(\mathcal{A})+\sum_{j=1}^{k} \mu_{P}^{\mathcal{C}_{j}^{P}}(\mathcal{A})
$$


From Theorem 2.4 we know that

$$
\mu_{P}^{1, \ldots, 1}(\mathcal{A})=\frac{1}{2} \sum_{j=1}^{k} n_{j}\left(E_{j}+1\right)-1-2 \omega- \begin{cases}0, & \text { if } \sum_{j=1}^{k} n_{j} E_{j} \tan A_{j}>0 \\ 1, & \text { otherwise }\end{cases}
$$

From Lemma 4.5 and (1) of Lemma 4.4 we get

$$
\mu_{P}^{\mathcal{C}_{j}^{P}}(\mathcal{A})=\frac{1}{2}\left(n_{j}-1\right) \cdot\left(E_{j}+1\right)
$$

Summing all up, we obtain the desired formula.

\section{Orthogonality with respect to the Hessian form of the oriented area}

Let us remind that $\mathcal{C}_{n}$ is the configuration space of cyclic polygons with at least three different vertices (see (2.2)). First, we parametrise $\mathcal{C}_{n}$ smoothly. For this we introduce

$$
\mathcal{H}_{n}=\left\{\left(\theta_{1}, \ldots, \theta_{n}\right) \in\left(S^{1}\right)^{n}: \#\left\{\theta_{1}, \ldots, \theta_{n}\right\} \geq 3\right\} / S^{1}
$$

where $S^{1}$ acts on $\left(S^{1}\right)^{n}$ diagonally by rotations. Consider the following map

$$
\begin{aligned}
\widetilde{\varphi}:\left(\left(S^{1}\right)^{n} \backslash \text { Diag }\right) \times \mathbb{R}_{>0} & \rightarrow\left(\mathbb{R}^{2}\right)^{n} \backslash \text { Diag } \\
\left(\theta_{1}, \ldots, \theta_{n}, R\right) & \mapsto R \cdot\left(\left(\cos \theta_{1}, \sin \theta_{1}\right), \ldots,\left(\cos \theta_{n}, \sin \theta_{n}\right)\right) .
\end{aligned}
$$

Lemma 5.1. $\widetilde{\varphi}$ induces a diffeomorphism $\varphi: \mathcal{H}_{n} \times \mathbb{R}_{>0} \rightarrow \mathcal{C}_{n}$.

Proof. $\varphi$ is obviously a bijection, so the only thing we need to check is that the Jacobian of $\widetilde{\varphi}$ has rank $(n+1)$ at every point. In fact, it is just a statement of the form ' $S^{1} \times \mathbb{R}_{>0}$ is diffeomorphic to $\mathbb{R}^{2} \backslash\{0\}$ via polar coordinates', but we compute the Jacobian for the sake of completeness:

$$
\operatorname{Jac} \varphi=\left(\begin{array}{c}
\operatorname{Jac}_{1} \varphi \\
\vdots \\
\operatorname{Jac}_{n} \varphi \\
\operatorname{Jac}_{n+1} \varphi
\end{array}\right)=\left(\begin{array}{cccccc}
-R \sin \theta_{1} & R \cos \theta_{1} & 0 & \ldots & 0 & 0 \\
\vdots & \vdots & \vdots & \ddots & \vdots & \vdots \\
0 & 0 & 0 & \ldots & -R \sin \theta_{n} & R \cos \theta_{n} \\
\cos \theta_{1} & \sin \theta_{1} & \cos \theta_{2} & \ldots & \cos \theta_{n} & \sin \theta_{n}
\end{array}\right)
$$

The first $n$ rows are obviously linearly independent. Suppose one has $\operatorname{Jac}_{n+1} \varphi=$ $\lambda_{1} \operatorname{Jac}_{1} \varphi+\cdots+\lambda_{n} \operatorname{Jac}_{n} \varphi$. Then for any $i=1, \ldots, n$ one gets

$$
\left(\cos \theta_{i}, \sin \theta_{i}\right)=\lambda_{1}\left(-R \sin \theta_{i}, R \cos \theta_{i}\right)=\lambda_{1} R\left(\cos \left(\theta_{i}+\pi / 2\right), \cos \left(\theta_{i}+\pi / 2\right)\right),
$$

which implies $\lambda_{i}=0$, a contradiction.

We now provide local coordinates for $\mathcal{C}_{n}$.

Lemma 5.2. Let $P \in \mathcal{C}_{n}$ be an admissible non-bifurcating cyclic polygon with edge lengths $l_{1}, \ldots, l_{n}>0$. For $Q \in \mathcal{C}_{n}$ let $t_{i}(Q)=l_{i}(Q)-l_{i}$. Then $\left(t_{1}, \ldots, t_{n}\right)$ are smooth local coordinates for $\mathcal{C}_{n}$ around $P$. 
Proof. In view of Lemma 5.1 we just need to show that for

$$
\begin{aligned}
\psi: \mathcal{H}_{n} \times \mathbb{R}_{>0} & \rightarrow \mathbb{R}^{n} \\
\left(\theta_{1}, \ldots, \theta_{n}, R\right) & \mapsto R \cdot\left(\sqrt{2-2 \cos \left(\theta_{2}-\theta_{1}\right)}, \ldots, \sqrt{2-2 \cos \left(\theta_{1}-\theta_{n}\right)}\right)
\end{aligned}
$$

$\operatorname{Jac} \psi$ is of rank $n$ at points where $\theta_{1} \neq \theta_{2} \neq \cdots \neq \theta_{n} \neq \theta_{1}$. Indeed, Jac $\psi$ is

$$
\left(\begin{array}{ccccc}
\frac{R \sin \left(\theta_{1}-\theta_{2}\right)}{\sqrt{2-2 \cos \left(\theta_{1}-\theta_{2}\right)}} & 0 & \cdots & 0 & \frac{R \sin \left(\theta_{1}-\theta_{n}\right)}{\sqrt{2-2 \cos \left(\theta_{1}-\theta_{n}\right)}} \\
\frac{R \sin \left(\theta_{2}-\theta_{1}\right)}{\sqrt{2-2 \cos \left(\theta_{2}-\theta_{1}\right)}} & \frac{R \sin \left(\theta_{2}-\theta_{3}\right)}{\sqrt{2-2 \cos \left(\theta_{2}-\theta_{3}\right)}} & \cdots & 0 & 0 \\
\vdots & \vdots & \vdots & \ddots & \vdots \\
0 & 0 & \cdots & \frac{R \sin \left(\theta_{n-1}-\theta_{n}\right)}{\sqrt{2-2 \cos \left(\theta_{n-1}-\theta_{n}\right)}} & 0 \\
0 & 0 & \cdots & \frac{R \sin \left(\theta_{n}-\theta_{n-1}\right)}{\sqrt{2-2 \cos \left(\theta_{n}-\theta_{n-1}\right)}} & \frac{R \sin \left(\theta_{n}-\theta_{1}\right)}{\sqrt{2-2 \cos \left(\theta_{n}-\theta_{1}\right)}} \\
\sqrt{2-2 \cos \left(\theta_{2}-\theta_{1}\right)} & \sqrt{2-2 \cos \left(\theta_{3}-\theta_{2}\right)} & \cdots & \sqrt{2-2 \cos \left(\theta_{n}-\theta_{n-1}\right)} & \sqrt{2-2 \cos \left(\theta_{1}-\theta_{n}\right)}
\end{array}\right)
$$

Since $2\left(\theta_{i+1}-\theta_{i}\right) \neq 0$, all the entries are well-defined and non-zero. Consider a vanishing non-trivial linear combination of columns. The form of the first $n$ rows forces the coefficient at the $i$-th column to be equal (up to the common multiplier) to $\frac{\sqrt{2-2 \cos \left(\theta_{i}-\theta_{i+1}\right)}}{\sin \left(\theta_{i}-\theta_{i+1}\right)}$, but then for the last row we have

$$
0=\sum_{i=1}^{n} \frac{2-2 \cos \left(\theta_{i}-\theta_{i+1}\right)}{\sin \left(\theta_{i}-\theta_{i+1}\right)}=2 \sum_{i=1}^{n} \tan \left(\frac{\theta_{i}-\theta_{i+1}}{2}\right)
$$

which means exactly that $P$ is bifurcating and contradicts the assumptions of the lemma. Thus, Jac $\psi$ has rank $n$ as desired.

Proof of Lemma 4.3. To prove the first two claims let us note that smooth structures on $\mathcal{E}^{P}, \mathcal{C}^{P}$, and $\mathcal{M}_{s m}(N)$ come from the smooth structure on Poly $n=\left(\mathbb{R}^{2}\right)^{n}$. Thus, the first claim immediately follows from Lemma 3.3 , as the only cyclic polygon fitting into a straight line is a complete fold, which is not admissible. The dimension of $\mathcal{E}^{P}$ is computed according to (2) in Proposition 3.7. From Lemma 5.1 it follows that $\mathcal{C}_{n}$ around $P$ is a smooth submanifold in Poly $_{n} / \mathrm{IsO}_{+}$, and from Lemma 5.2 we deduce that $\mathcal{C}^{P}$ around $P$ is a smooth $(n-k)$-dimensional submanifold of $\mathcal{C}_{n}$ as it is a preimage of the linear subspace of codimension $k$ in $\mathbb{R}^{n}$ under the map $Q \mapsto\left(t_{1}(Q), \ldots, t_{n}(Q)\right)$. Thus the second claim is also proved.

The third claim is equivalent (by dimension count) to representability of every vector in $T_{P} \mathcal{M}_{s m}(\mathbf{N})$ as a sum of two vectors from $T_{P} \mathcal{E}^{P}$ and $T_{P} \mathcal{C}^{P}$ respectively, but this is indeed the case since every polygon $Q$ near $P$ in $\mathcal{M}_{s m}(\mathbf{N})$ can be obtained first by a move in $\mathcal{C}^{P}$ making the edges of desired length (by Lemma 5.2) and then by a move inside $\mathcal{E}^{Q}$. 
Finally, we establish the fourth claim. Consider $v \in T_{P} C$ and $w \in T_{P} E$. To compute $\operatorname{Hess}_{P} \mathcal{A}(v, w)$, we choose a curve $\gamma:(-\varepsilon, \varepsilon) \rightarrow T_{P} C$ such that $\gamma(0)=P$ and $\gamma^{\prime}(0)=v$, then we extend $w$ to a vector field $W(t) \in T_{\gamma(t)} E^{\gamma(t)}$ along $\gamma$. Then

$$
\operatorname{Hess}_{P} \mathcal{A}(v, w)=\left.\frac{d}{d t}\right|_{t=0} d_{\gamma(t)} \mathcal{A}(W(t)) .
$$

But $d_{\gamma(t)} \mathcal{A}$ vanishes on $T_{\gamma(t)} E^{\gamma(t)}$ by Theorem 4.1 .

The following lemma allows one to relate $\mathcal{C}_{j}^{P}$ with $\mathcal{C}^{P}$.

Lemma 5.3. Let $P \in \mathcal{C}_{n}$ be an admissible non-bifurcating cyclic polygon such that $l_{1}=l_{2}$ and $\angle p_{1} o p_{2}=\angle p_{2} o p_{3}$, where $o$ is the centre of the circumscribed circle $\Omega$. Let $V$ be $a$ local vector field around $P$ equal to $\left(\frac{\partial}{\partial t_{1}}-\frac{\partial}{\partial t_{2}}\right)$ in the coordinates from Lemma 5.2. Then $(V R)(P)=0$ and $\left(V d_{a b}\right)(P)=0$ for $a, b \in\{1, \ldots, n\} \backslash\{2\}$, where $V f$ is the derivative of a function $f$ along $V, R(Q)$ is the radius of the circumscribed circle of $Q$ and $d_{a b}(Q)=\left|q_{b}-q_{a}\right|$.

Proof. Consider a curve $P(s):(-\varepsilon, \varepsilon) \rightarrow \mathcal{C}_{n}, \quad\left(t_{1}, \ldots, t_{n}\right)(P(s))=(s,-s, 0, \ldots, 0)$. We choose representatives $\widetilde{P}(s) \in$ Poly $_{n}$ in such a way that $o_{\widetilde{P}(s)}=(0,0)$ and $\left(p_{3}-p_{1}\right)$ is codirectional with $x$-axes. Notice that $\widetilde{P}(-s)$ is obtained from $\widetilde{P}(s)$ by the following procedure: $p_{i}(-s)=p_{i}(s)$ for $i \neq 2$ and $p_{2}(-s)$ is symmetric to $p_{2}(s)$ relative to $y$-axes. From this it follows that $\widetilde{P}(s)-\widetilde{P}(-s))=(0,0,2 \eta, 0, \ldots, 0)$ for some $\eta>0$. Hence all $p_{i}$ for $i \neq 2$ are not moving in the first approximation, which implies the statement of the lemma.

Proof of Lemma 4.4. The space $\left\{Q \in \mathcal{M}_{s m}(\mathbf{N}): q_{i}=p_{i}\right.$ for $\left.i \notin j^{*} \backslash\{s(j)\}\right\} \quad$ is a smooth submanifold in $\mathcal{M}_{s m}(\mathbf{N})$ diffeomorphic to $\mathcal{M}_{s m}\left(\left(n_{j}, L_{j}\right),\left(1,\left|p_{s(j+1)}-p_{s(j)}\right|\right)\right)$. Under this identification, $\mathcal{C}_{j}^{P}$ is just $\mathcal{C}^{P}$. Applying (2) of Lemma 4.3 to $\mathcal{M}_{s m}\left(\left(n_{j}, L_{j}\right),\left(1,\left|p_{s(j+1)}-p_{s(j)}\right|\right)\right)$, we get the first claim.

To establish the second claim it suffices to find bases in every $T_{P} \mathcal{C}_{j}^{P}$ such that their disjoint union forms a basis of $T_{P} \mathcal{C}^{P}$. Consider the coordinates from Lemma 5.2. On the one hand, when we consider cyclic polygons coordinatised by $\left(t_{1}, \ldots, t_{n}\right)$, the vectors $\left(\frac{\partial}{\partial t_{i-1}}-\frac{\partial}{\partial t_{i}}\right)$ for inner $i$ form a basis of $T_{P} \mathcal{C}^{P}$. On the other hand, when we consider $\mathcal{C}_{j}^{P}$ coordinatised by $\left(s_{i}\right)_{i \in j^{*} \backslash\{s(j)\}}$, where $s_{i}=l_{i}(Q)-l_{i}(P)$, the vectors $\left(\frac{\partial}{\partial s_{i-1}}-\frac{\partial}{\partial s_{i}}\right)$ for $i \in j^{*} \backslash\{s(j)\}$ form a basis of $T_{P} \mathcal{C}_{j}^{P}$. It now follows from Lemma 5.3 that $\left(\frac{\partial}{\partial t_{i-1}}-\frac{\partial}{\partial t_{i}}\right)=$ $\left(\frac{\partial}{\partial s_{i-1}}-\frac{\partial}{\partial s_{i}}\right)$ for $i \in j^{*} \backslash\{s(j)\}$ thus the second claim is proven.

Now we pass to the third claim. Consider $v \in T_{P} \mathcal{C}_{j}^{P}$ and $w \in T_{P} \mathcal{C}_{h}^{P}$, take a curve $\gamma:(-\varepsilon, \varepsilon) \rightarrow \mathcal{C}_{j}^{P}$ such that $\gamma(0)=P$ and $\gamma^{\prime}(0)=v$, and a curve $\sigma:(-\varepsilon, \varepsilon) \rightarrow \mathcal{C}_{h}^{P}$, such that $\sigma(0)=P$ and $\sigma^{\prime}(0)=w$. Then extend $w$ to a vector field $W(t) \in T_{\gamma(t)} \mathcal{M}_{s m}(\mathbf{N})$ along $\gamma$ by setting $W(t)=\sigma_{t}^{\prime}(0)$, where $\sigma_{t}:(-\varepsilon, \varepsilon) \rightarrow \mathcal{C}_{h}^{\gamma(t)}$ is such that $\sigma_{t}(0)=\gamma(t)$ and for all $i \in j^{*} \backslash s(j)$ the $i$-th vertex of $\sigma_{t}(s)$ is the same as the $i-t h$ vertex of $\sigma(s)$. Then

$$
\operatorname{Hess}_{P} \mathcal{A}(v, w)=\left.\frac{d}{d t}\right|_{t=0} W(t) \mathcal{A},
$$

and it vanishes since $W(t) \mathcal{A}$ does not depend on $t$. 


\section{Configuration spaces of polygons with perimeter and one edge length fixed}

These are the spaces $\mathcal{M}((n, L),(1, l))$ for $L \geq l$. Vividly speaking, it is the space of broken lines of given length with fixed endpoints. One can think that the first and the last vertices have coordinates $(0,0)$ and $(l, 0)$ respectively. Our interest in these spaces was first motivated by the fact that they are simple enough to be studied completely, but then it turned out that they are important for understanding the case of general necklaces.

Proposition 6.1 (Configuration space in the 'two consecutive beads are fixed' case). Let $L>l$ and $n \geq 2$. Then $\mathcal{M}((n, L),(1, l))$ is homeomorphic to the sphere $S^{2 n-3}$.

Proof. By setting $p_{1}=(0,0)$ and $p_{n+1}=(l, 0)$ we identify $\mathcal{M}((n, L),(1, l))$ with the level set

$$
\begin{aligned}
F^{-1}(L) & =\left\{\left(p_{2}, \ldots, p_{n}\right) \in\left(\mathbb{R}^{2}\right)^{n-1}: F\left(p_{2}, \ldots, p_{n}\right)=L\right\}, \\
\text { where } \quad F\left(p_{2}, \ldots, p_{n}\right) & =\left|p_{2}\right|+\left|p_{3}-p_{2}\right|+\cdots+\left|p_{n}-p_{n-1}\right|+\left|(l, 0)-p_{n}\right| .
\end{aligned}
$$

$F$ is a convex function as sum of convex functions. The sublevel set $F^{-1}((-\infty, L])$ is bounded since if any of $\left|p_{i}\right|$ is greater than $L$, then $F\left(p_{2}, \ldots, p_{n}\right) \geq L$ by triangle inequality. Also, the set $F^{-1}((-\infty, L))$ is non-empty, since if all of the $p_{i}$ are in the disk of radius $\delta$ around $(l / 2,0)$, then $F\left(p_{2}, \ldots, p_{n}\right)<(l / 2+\delta)+(n-3) \delta+(l / 2+\delta)=l+(n-1) \delta$, which is less than $L$ for small $\delta$. So, $F^{-1}(L)$ is a boundary of the compact convex set $F^{-1}((-\infty, L]) \subset\left(\mathbb{R}^{2}\right)^{n-1}$ with non-empty interior and thus is homeomorphic to $S^{2 n-3}$.

The following two propositions are easily deduced from Theorems 4.1 and 4.2 respectively.

Proposition 6.2 (Critical points in the 'two consecutive beads are fixed' case). Let $L>l$ and $n \geq 2$. Then critical points of $\mathcal{A}$ on $\mathcal{M}_{s m}((n, L),(1, l))$ are in bijection with the solutions of

$$
\left|U_{n-1}(x)\right|=\frac{n l}{L}
$$

where $U_{n-1}$ is the $(n-1)$-th Chebyshev polynomial of second kind, that is, $U_{n-1}(\cos \varphi)=\frac{\sin n \varphi}{\sin \varphi}$.

Proof. By Theorem 4.1 a configuration $P \in \mathcal{M}_{s m}((n, L),(1, l))$ is a critical point of $\mathcal{A}$ if and only if it is inscribed in a circle $\Omega$ with centre $o$ and radius $R$ in such a way that $\angle p_{1} o p_{2}=\cdots=\angle p_{n} o p_{n+1}=: \alpha(P)=\alpha$. Let us construct a bijection

$\left\{\right.$ critical points of $\mathcal{A}$ on $\left.\mathcal{M}_{s m}((n, L),(1, l))\right\} \rightarrow\{$ solutions of $(6.1)\}, P \mapsto c_{P}$.

Let $c_{P}=\cos (\alpha / 2)$, where $\alpha / 2 \in(0, \pi)$. Since $L / n=R \sqrt{2-2 \cos \alpha}=2 R \sin (\alpha / 2)$ and $l=R \sqrt{2-2 \cos (n \alpha)}=2 R|\sin (n \alpha / 2)|$, we get $U_{n-1}\left(c_{P}\right)=n l / L$. Since the map

$$
\frac{\mathbb{R}}{2 \pi \mathbb{Z}} \backslash\{0\} \rightarrow(-1,1), \alpha \mapsto \cos (\alpha / 2)
$$

is a bijection and $P$ is uniquely determined by $\alpha(P)$, the constructed map $P \mapsto c_{P}$ is indeed a bijection. 
Proposition 6.3 (Morse index in the 'two consecutive beads are fixed' case). If $P$ is an admissible non-bifurcating critical configuration of $\mathcal{A}$ on $\mathcal{M}_{\text {sm }}((n, L),(1, l))$, then its Morse index is

$$
\mu_{P}^{n, 1}(\mathcal{A})= \begin{cases}2 n-2-i, & \text { if } c_{P} \text { is the } i \text {-th largest positive solution of }(6.1) \\ i-1, & \text { if } c_{P} \text { is the } i \text {-th smallest negative solution of }(6.1)\end{cases}
$$

Proof. By symmetry reasons, to prove the claim, it suffices to prove it only for $P$ with $c_{P}>0$. Then by Theorem 4.2 one has

$\mu_{P}^{n, 1}(\mathcal{A})=(2 n-1)+\frac{1}{2}\left(\varepsilon_{n+1}+1\right)-1-w_{P}- \begin{cases}0, & \text { if } n \tan (\alpha / 2)>\varepsilon_{n+1} \tan (n \alpha / 2) ; \\ 1, & \text { otherwise. }\end{cases}$

The roots and extrema of $U_{n-1}(t)$ are interchanging. Let us start from $t=1$ and move to the right. The extrema correspond to the bifurcating polygons (i.e. those with $n \tan (\alpha / 2)=$ $\left.\varepsilon_{n+1} \tan (n \alpha / 2)\right)$ and the roots correspond to polygons with $l_{n+1}=0$. So, when $t$ passes a root, $\varepsilon_{n+1}$ changes from 1 to -1 and whenever $t$ passes an extrema, the last summand changes from 0 to 1 . When $p_{1} p_{n+1}$ passes through $o, w_{P}$ increases by 1 , and $\varepsilon_{n+1}$ changes from -1 to 1 , which does not change the Morse index. The right-most $t$ corresponds to the global maximum, so the above argument completes the prove.

Finally, we check the last yet unproven ingredient in the proof of Theorem 4.2.

Proof of Lemma 4.5. Let $P$ be as in the lemma. Without loss of generality we can assume that $\Omega_{P}=\Omega$ is the unit circle with centre $o$, and, due to symmetry, it is enough to prove the statement for $P$ with $w_{P}>0$. We should prove that the function

$$
\frac{\mathcal{A}}{l_{n+1}^{2}}:\left\{\text { polygons } P \text { inscribed in the unit circle with } \frac{\mathcal{L}_{1}(P)}{l_{n+1}(P)}=\frac{L}{l}\right\} \rightarrow \mathbb{R}
$$

attains a non-degenerate local maximum at $P$. For this it suffice to prove that the function

$$
\begin{aligned}
& G:\left\{\begin{array}{l}
\text { polygons inscribed } \\
\text { in the unit circle }
\end{array}\right\} \rightarrow \mathbb{R} \\
& G(Q)=\frac{2 \mathcal{A}(Q)}{l_{n+1}(Q)^{2}}-\lambda\left(\frac{\mathcal{L}_{1}(Q)^{2}}{l_{n+1}(Q)^{2}}-\frac{L^{2}}{l^{2}}\right)-\mu\left(\frac{\mathcal{L}_{1}(Q)^{2}}{l_{n+1}(Q)^{2}}-\frac{L^{2}}{l^{2}}\right)^{2}
\end{aligned}
$$

attains a non-degenerate local maximum at $P$ for suitable $\lambda$ and $\mu$. Set $\alpha=\angle p_{1} o p_{2}=\cdots=\angle p_{n} o p_{n+1} \in(0, \pi)$ and introduce local coordinates by setting $t_{i}(Q)=\angle q_{i} o q_{i+1}-\alpha$ for $i=1, \ldots, n$. First, we write the functions involved in the definition (6.2) in these coordinates:

$$
\begin{aligned}
l_{n+1}\left(t_{1}, \ldots, t_{n}\right) & =\sqrt{2-2 \cos \left(n \alpha+\sum_{i=1}^{n} t_{i}\right)} \\
\mathcal{L}_{1}\left(t_{1}, \ldots, t_{n}\right) & =\sum_{i=1}^{n} \sqrt{2-2 \cos \left(\alpha+t_{i}\right)} ; \\
2 \mathcal{A}\left(t_{1}, \ldots, t_{n}\right) & =\sum_{i=1}^{n} \sin \left(\alpha+t_{i}\right)-\sin \left(n \alpha+\sum_{i=1}^{n} t_{i}\right) .
\end{aligned}
$$


Secondly, we perform the computations in the 2 -jets at the point $P$, which by the aforementioned coordinates are identified with $\mathbb{R}\left[t_{1}, \ldots, t_{n}\right] / I$, where $I$ is the ideal generated by all products $t_{i} t_{j} t_{h}$ with $i, j, h=1, \ldots, n$. It turns out that the 2 -jets of the functions we are interested in are all contained in the subring $\mathbb{R}+\mathbb{R} T_{1}+\mathbb{R} T_{1}^{2}+\mathbb{R} T_{2}$, where $T_{1}=\sum_{i=1}^{n} t_{i}$ and $T_{2}=\sum_{i=1}^{n} t_{i}^{2}$. This subring is naturally identified with the ring $\mathbb{R}\left[T_{1}, T_{2}\right] /\left(T_{1}^{3}, T_{2}^{2}, T_{1} T_{2}\right)$. With all the identifications done, the 2 -jets of the functions involved in the definition (6.2) look as follows:

$$
\begin{aligned}
j_{2} l_{n+1} & =l \cdot\left(1+\frac{1}{2} \cot \left(\frac{n \alpha}{2}\right) T_{1}-\frac{1}{8} T_{1}^{2}\right) ; \\
j_{2} \mathcal{L}_{1} & =L \cdot\left(1+\frac{1}{2 n} \cot \left(\frac{\alpha}{2}\right) T_{1}-\frac{1}{8 n} T_{2}\right) ; \\
j_{2}(2 \mathcal{A}) & =(n \sin \alpha-\sin (n \alpha))+(\cos \alpha-\cos n \alpha) T_{1}-\frac{\sin \alpha}{2} T_{2}+\frac{\sin (n \alpha)}{2} T_{1}^{2} .
\end{aligned}
$$

Now, setting $x=\tan \frac{\alpha}{2}$ and $y=\tan \frac{n \alpha}{2}$, we can write the 2 -jets of the summands in (6.2) in more or less compact form:

$$
\begin{aligned}
& j_{2}\left(\frac{\mathcal{L}_{1}^{2}}{l_{n+1}^{2}}-\frac{L^{2}}{l^{2}}\right)=\frac{n x\left(1+y^{2}\right)(y-n x)}{y^{3}\left(1+x^{2}\right)} T_{1}-\frac{n x^{2}\left(1+y^{2}\right)}{4 y^{2}\left(1+x^{2}\right)} T_{2}+C_{1}(n, x, y) T_{1}^{2} ; \\
& j_{2}\left(\frac{\mathcal{L}_{1}^{2}}{l_{n+1}^{2}}-\frac{L^{2}}{l^{2}}\right)^{2}=\frac{n^{2} x^{2}\left(1+y^{2}\right)^{2}(y-n x)^{2}}{y^{6}\left(1+x^{2}\right)} T_{1}^{2} ; \\
& j_{2}\left(\frac{2 \mathcal{A}}{l_{n+1}^{2}}-\frac{2 \mathcal{A}(P)}{l^{2}}\right)=\frac{\left(1+y^{2}\right)(y-n x)}{2 y^{3}\left(1+x^{2}\right)} T_{1}-\frac{x\left(1+y^{2}\right)}{4 y^{2}\left(1+x^{2}\right)} T_{2}+C_{2}(n, x, y) T_{1}^{2} .
\end{aligned}
$$

To get rid of $T_{1}$ in $j_{2} G$ we set $\lambda=\frac{1}{2 n x}$, and then finally obtain

$$
\begin{aligned}
j_{2}(G-G(P)) & =-\frac{x\left(1+y^{2}\right)}{8 y^{2}\left(1+x^{2}\right)} T_{2} \\
& +\left(C_{2}(n, x, y)-\frac{1}{2 n x} C_{1}(n, x, y)-\mu \cdot \frac{n^{2} x^{2}\left(1+y^{2}\right)^{2}(y-n x)^{2}}{y^{6}\left(1+x^{2}\right)}\right) T_{1}^{2} .
\end{aligned}
$$

Note that the first summand is a negative definite quadratic form since $x>0$. As for the second one, $n x-y \neq 0$ as $P$ is not bifurcating, and thus, whatever $C_{1}$ and $C_{2}$ are, when $\mu$ is big enough the second term is a non-positive definite quadratic form. Therefore, $G$ attains a non-degenerate local maximum at $P$ for $\lambda=\frac{1}{2 n x}$ and large positive $\mu$.

\section{ORCID iD}

Daniil Mamaev (D) https://orcid.org/0000-0002-7606-4276

\section{References}

[1] R. Connelly and E. D. Demaine, Geometry and topology of polygonal linkages, in: C. D. Toth, J. O'Rourke and J. E. Goodman (eds.), Handbook of Discrete and Computational Geometry, CRC Press, Boca Raton, Florida, pp. 233-256, 2017, https: / /www.taylorfrancis. com/books/e/9781315119601/chapters/10.1201/9781315119601-9.

[2] M. Farber, Invitation to Topological Robotics, Zurich Lectures in Advanced Mathematics, European Mathematical Society (EMS), Zürich, 2008, doi:10.4171/054. 
[3] J. Gordon, G. Panina and Y. Teplitskaya, Polygons with prescribed edge slopes: configuration space and extremal points of perimeter, Beitr. Algebra Geom. 60 (2019), 1-15, doi:10.1007/ s13366-018-0409-3.

[4] M. Kapovich and J. Millson, On the moduli space of polygons in the euclidean plane, J. Differential Geom. 42 (1995), 430-464, doi:10.4310/jdg/1214457034.

[5] G. Khimshiashvili and G. Panina, Cyclic polygons are critical points of area, Zap. Nauchn. Sem. S.-Peterburg. Otdel. Mat. Inst. Steklov. (POMI) 360 (2008), 238-245, doi:10.1007/ s10958-009-9417-z.

[6] G. Khimshiashvili and G. Panina, On the area of a polygonal linkage, Dokl. Akad. Nauk. Math. 85 (2012), 120-121, doi:10.1134/s1064562412010401.

[7] G. Khimshiashvili, G. Panina and D. Siersma, Extremal areas of polygons with fixed perimeter, Zap. Nauchn. Sem. S.-Peterburg. Otdel. Mat. Inst. Steklov. (POMI) 481 (2019), 136-145, doi: 10.1007/s10958-020-04835-9.

[8] J. C. Leger, Aire, périmètre et polygones cocycliques, 2018, arXiv:1805.05423 [math. CO].

[9] G. Panina and A. Zhukova, Morse index of a cyclic polygon, Cent. Eur. J. Math. 9 (2011), 364-377, doi:10.2478/s11533-011-0011-5.

[10] D. Siersma, Extremal areas of polygons, sliding along a circle, 2020, arXiv:2001.10882 [math.CO].

[11] A. Zhukova, Morse index of a cyclic polygon II, St. Petersburg Math. J. 24 (2013), 461-474, doi:10.1090/s1061-0022-2013-01247-7. 